who had developed photosensitivity insisted on continuing treatment rather than sacrifice pain relief or mobility.

The 61 deaths reported to the Committee on Safety of Medicines may underrepresent the mortality attributable to benoxaprofen but, if the widely quoted estimate that more than half a million patients have received it is correct, it is likely that at least 10000 patients died from natural causes while taking benoxaprofen. The deaths attributed to benoxaprofen probably account for only a very small fraction of the total mortality in this predominantly elderly population.

We had already commenced a new and larger study of benoxaprofen designed to test whether or not the new instructions about the treatment of elderly patients had been effective. Two antiarthritic agents not previously included in prescription-event monitoring had been selected as controls. The suspension of the licence for benoxaprofen halted this study, but, fortunately, we had already identified a large number of patients who started treatment last year. Less than $10 \%$ of them had been included in the pilot study, and we hope soon to be able to test various hypotheses in a large: population.

To avoid disturbing the progress of 10 other drugs currently in the prescription-event monitoring pipeline we propose to introduce the expanded benoxaprofen study in one region at a time. This is not merely an academic exercise. It is as important to determine the characteristics (for example, age or diagnosis) of patients for whom the benefits may outweigh the risks as it is to confirm the extent of the risk in vulnerable groups.

I do hope that we can count on your help in this extension of the benoxaprofen study and in other prescription-event monitoring studies. Once again I would like to thank several thousand colleagues who have already helped in the pilot study.

W $\mathrm{H}$ W INMAN

Drug Surveillance Research Unit, University of Southampton, Southampton SO2 3FL

\section{Chest radiography as a marker of alcoholism}

SIR,-Further to the findings of Dr D R M Lindsell and others (28 August-4 September, p 597) I would like to furnish the following data. I have described the medical morbidity of 235 consecutive admissions to a detoxification centre, ${ }^{1}$ and part of that study involved a chest $x$-ray examination and serum liver function tests. The detoxification centre at the University Hospital of South Manchester was opened in October 1977. A man or woman found by the police to be "drunk and incapable" or "drunk and disorderly" and known to have had similar convictions in the past could be taken by a police officer directly to the detoxification centre. Once there, all criminal charges were dropped, and the patient was immediately examined by a doctor. Of the 235 chest radiographs taken, 24 showed rib fractures and four showed clavicular fractures, giving a total of 28 (or $12 \%$ ). Only one patient had any disturbance of liver function tests.

These findings may indicate that in a population of "binge" drinkers the periods of sobriety enforced either by imprisonment or by poverty serve as a protective mechanism against the development of liver damage, which often occurs in the "chronic imbibers." These results also confirm that fractures on a chest radiograph are still more common in any population of problem drinkers than in the normal population, even in the absence of liver disease.
The drinking pattern of the group I studied was loss of control "binge" drinking, which inevitably led to drunkenness. It was felt that the fractures occurred due to injuries sustained while drinking, and this was supported by discussions with the individuals.

\section{A D REDMOND}

Intensive Care Unit,

Royal Preston Hospital,

Preston PR2 4HT

${ }^{1}$ Redmond AD. The medical morbidity of 235 individuals admitted to Britain's first hospital based, purposebuilt, detoxification centre. Manchester: Victoria

\section{Diabetic complications: retinopathy}

SIR,-While I wholeheartedly agree with $\mathrm{Dr}$ Peter Watkins (7 August, p 425) when he says that diabetic retinopathy needs to be actively sought if it is to be detected early enough to prevent blindness, I would take issue with his further statement that diabetic maculopathy should be treated when visual acuity begins to decline-that is, a decrease of two lines on the Snellen chart. This seems to me to be rather closing the stable door after the horse has bolted.

The British multicentre photocoagulation trial showed that the patients who do best from treatment with photocoagulation in relation to maculopathy are those with exudates encroaching on the macula but in whom the vision remains at $6 / 5$ or $6 / 6$. These patients had one eye treated and the other eye was left untreated and there were four further lines of deterioration in the untreated eye compared with the treated eye over five years. If one waits until these exudates have reduced the vision to $6 / 12$ or $6 / 18$ then the difference in deterioration between the two eyes is reduced to the order of two lines over five years. This evidence therefore suggests that exudative maculopathy should certainly be treated in the premaculopathy phase. In addition, I have noticed as a complication of treatment that hard exudates can enlarge immediately after treatment. If one waits until the hard exudates are poised on the edge of the macula there is therefore a greater risk of morbidity from treatment-another indication for earlier treatment.

It is essential that a situation is created in which the retinas of people suffering from diabetes mellitus are examined at regular intervals, especially once they are into the danger zone for diabetic retinopathy chronologically, so that their retinal lesions may be treated before they become symptomatic. It is often too late to treat disc new vessels once the symptoms of vitreous haemorrhage have begun to develop or maculopathy once the symptoms of reduced central visual acuity have begun to develop. This is because a patient may have the most severe form of proliferative retinopathy or the most devastating hard exudates poised on the edge of the fovea without having any symptoms or being aware of any serious problem.

C TOWNSEND

Western Ophthalmic Hospital,

Western Ophthal

\section{ABC of Diabetes: diabetic emergencies}

SIR,-I refer to the letter of Dr O M P Jolobe (14 August, p 509) and his suggested programme for managing diabetic emergencies.
The point that insulin should be withheld until the potassium result is known is well made, but he loses my support with his "counsel of safety."

My point is that, although probably merely an oversight, it is not specified in the letter which diabetic emergency one is dealing with. Should the stated "counsel of safety" happen to be followed in a patient in a hyperosmolar, non-ketotic state the resulting hypernatraemia might be disastrous-rather more disastrous and irreversible than the hypokalaemia he fears. Especially so if one is prepared to follow Dr Watkins' regimen for the stated two hours while waiting for the electrolyte results to be phoned through, by which time two to three litres of normal saline may have been infused.

The diagnosis of the hyperosmolar aketotic state is not difficult-once it has been thought of. With dehydration making urine unavailable for testing and plasma ketostix testing by no means universal, the diagnosis might well be overlooked by the house physician.

None of the above, of course, is earthshattering information, but I would not want a newly qualified colleague to come away from Dr Jolobe's letter with the idea that a fast saline infusion may be given with impunity to any patient with "diabetic precoma."

In practice it is unusual for the biochemist to take two hours to give the results of blood sugar, electrolytes, or even blood gas estimations. Since it seems to me that this length of time would be unlikely to be critical in the outcome, if one is to wait for two hours a safer counsel of safety might be to do absolutely nothing in the meantime.

Paul Baker

Warrington General Hospital Warrington WA5 1QG

SIR,-Discussing with colleagues here the timing of insulin and potassium in diabetic ketoacidosis (14 August, p 509), I agree that two hours is too long to wait for a potassium result before commencing insulin. Because the vast majority of diabetics in ketoacidosis will have levels of serum potassium verging on hyperkalaemia, it is safer to commence insulin without waiting for the potassium result if one expects laboratory delays.

O M P Jolobe

Dudley Road Hospital,
Birmingham B18 7QH

\section{The arms race and health care}

SIR,-Dr D J Holdstock (7 August, p 421) has indicated the scale of current diversion of resources into armaments. A proportion of that expenditure is devoted to weapons which if used are likely to cause very extensive civilian mortality and morbidity. The BMA Board of Science will provide next year an assessment of these possible effects, but it has not been asked to comment on the ethical aspects of supporting use of weapons with mass destructive capability developed as a result of technological progress in the last few decades. In view of the nature of the destructive effects of such weapons, these ethical issues require adequate evaluation by the profession.

Although wars in the past often had adverse and at times devastating effects on civilians, in general it could have been argued that maintaining the integrity of a nation state and safeguarding its resources by armed conflict served the interests 